\title{
Dielectric and dynamic-mechanical study of the mobility of poly(t-butylacrylate) chains in diblock copolymers: Polystyrene-b-poly(t-butylacrylate)
}

\author{
Mario Encinar , Eduardo Guzmán , Margarita G. Prolongo , Ramón G. Rubio ， \\ Claudia Sandoval , Fernando González-Nilo , Ligia Gargallo , Deodato Radić \\ Departamento de Química Física I, Facultad Química, Universidad Complutense, 28040-Madrid, Spain \\ Departamento de Materiales y Producción Aeroespacial, ETSI Aeronáuticos, Universidad Politécnica, 28040-Madrid, Spain \\ Departamento de Ouímica Física, Facultad de Química, Pontificia Universidad Católica de Chile, Vicuña Mackenna 4860, Casilla 306, Santiago 22, Chile \\ Centro de Bioinformática y Simulación Molecular (CBSM), Universidad de Talca, 2 Norte 685, Casilla 721, Talca, Chile
}

Keywords:

Dielectric relaxation

Block copolymers

Glass transition

\begin{abstract}
A B S T R A C T
A calorimetric, dielectric and dynamic-mechanical study of the dynamics of the poly(t-butyl acrylate) (PtBa) chains has been carried out in a PtBa homopolymer and two polystyrene (PS)-b-PtBa block copolymers with different PtBa chain lengths. The DSC results show that the size of the cooperative rearranging regions is similar in the homopolymers and the copolymers, both for the PtBa rich- and the PS-rich regions. Therefore, no significant contributions are found arising from composition fluctuations in the copolymers. The relaxation map obtained from dielectric relaxation indicates that there are no differences in the temperature dependence of the $\alpha$-relaxation of the PtBa block in the three samples studied. However, there are larger differences for the values obtained from DMTA experiments. Contrary to the $\alpha$-relaxation, the relaxation map for the $\beta$-transition shows that the characteristic times for the PtBa blocks are smaller in the homopolymer than in the copolymers. In principle, these are unexpected results because the $\beta$-relaxations have a more local character than the $\alpha$-ones. The width of the $\alpha$-relaxation increases with $T$ for all the samples, and it is slightly larger for the copolymers. The intensity of the $\alpha$-relaxation is larger (between 3 and 4 times) for the homopolymer. Considering the molecular weights of the PtBa blocks, this effect has to be ascribed to the existence of frozen amorphous PtBa due to the existence of the glassy PS domains in the microphase separated copolymers.

Molecular Dynamic Simulations (MDSs) for different sequences of the polymers under study were carried out. The conformational analysis was carried out between 1000 and $1700 \mathrm{~K}$. The analysis of the variation of angles $\phi_{1}$ and $\phi_{2}$ of the ester group of PtBa points out the existence of a correlation between the conformational changes of the side group of the polymer chains and their relaxational behaviour.
\end{abstract}

\section{Introduction}

Block copolymers have attracted much attention due to their tendency to form ordered structures at the nanometer scale below the so-called order-disorder temperature $T_{\mathrm{OD}}$ The equilibrium thermodynamic state of an $\mathrm{A}-\mathrm{B}$ diblock copolymer melt is determined by $\chi N$ and the composition $f=N_{\mathrm{A}} / N$, where $N_{\mathrm{A}}$ is the number of segments of type $\mathrm{A}$ in the chain, $N$ is the total number of segments in the copolymer chain, and $\chi$ is Flory's interaction parameter between the two types of segments ( $\chi$ depends on $1 / T, T$ being the temperature). For values of $\chi N$ greater than a critical value $(\chi N)_{c}$, a microphase separation occurs, and different microdomains are formed depending on the value of $f$. Dispersion of spheres or cylinders of one of the blocks in a continuous matrix of the other block, lamellar and bicontinuous phases has been described.

When both blocks have well separated values of the glass transition temperature $T_{\mathrm{g}}$, the dynamics of low- $T_{\mathrm{g}}$ blocks can be affected by the presence of a rigid phase, thus being different from the corresponding homopolymer. Kotaka and Adachi have found that the dynamics of the normal and segmental modes of poly(isoprene) blocks (PI) in PI-b-PS and PI-b-PS-b-PI copolymers (PS referring to polystyrene) is strongly affected by the morphology of the samples. Moreover, for $T<T_{g}$ (PS), they concluded that the interchain cooperativity increases. Alig et al. found that the 
spectrum of relaxation times was significantly broader in PS-b-PI at $T<T_{\mathrm{g}}(\mathrm{PS})$, than in the corresponding homopolymers. Also at $T>T_{\text {ODT }}$ the existence of concentration fluctuations in the copolymers leads to two distinct primary relaxation processes. Karatasos also studied PI-b-PS copolymers and described a relaxation process at $T<T_{\mathrm{g}}$ (PS) associated to the chains tethered to the interfaces between the PI and the PS phases. Similar conclusions were reached by Kyritsis et al. by Vogt et al. by Moreno and Rubio and by Ma et al. for other block copolymers.

The analysis of the dielectric relaxation of the block copolymers studied is complicated by the partial overlapping of the segmental and normal modes, or by the interfacial effects arising from the existence of a semicrystalline block. In addition, simultaneous analysis of dielectric relaxation (DR) and dynamicmechanical spectroscopy (DMTA) results for block copolymers is rather scarce. In the present work we have selected two copolymers of poly(t-butyl acrylate) (PtBa) and PS. These two blocks are amorphous, immiscible, and they have well separated $T_{\mathrm{g}}$ s. These block copolymers are in the phase separated state, in the strong segregation limit. As in the PI-b-PS copolymer, only one of the blocks (the PtBa one) is dielectrically active. However, the PtBa block has no dipole moment along the chain, thus no normal mode is expected to exist, and therefore the segmental mode should not be affected by other dynamic contributions. The results obtained with the copolymers will be compared with those of the PtBa homopolymer. On the other hand, both blocks are expected to be active in dynamic-mechanical spectroscopy, thus allowing a comparison of the results obtained by both techniques.

The DMTA results will show that the copolymers behave as thermorheologically simple materials, allowing one to build master curves for the relaxation of both the PtBa and the PS blocks. However, the DR results show a more complicated scenario, since the shape of the relaxation curves has different temperature dependencies in the copolymers and in the PtBa homopolymer.

A surprising result is that while the relaxation map of the $\alpha$ mode of the PtBa block is almost the same in the copolymers than in the homopolymer, the relaxation times of the $\beta$-mode are different. This behaviour, although somewhat surprising in view of the local character of the $\beta$-mode, can be explained by the computer simulation results.

The intensity of the $\alpha$-relaxation of the PtBa blocks, measured by dielectric relaxation, is much smaller than that for the PtBa homopolymer. This suggests the existence of frozen amorphous PtBa material due to the existence of the glassy PS regions in the microphase separated block copolymers.

\section{Experimental}

The copolymers were obtained from Polymer Source (Canada). The molecular weights of the PtBa and PS blocks are given in Table 1. Size Exclusion Chromatography (SEC) using tetrahydrofuran as solvent allowed us to determine the overall molecular weight and the polydispersity index $M_{\mathrm{w}} / M_{\mathrm{n}}$; the relative content of the co-monomers was obtained by ${ }^{13} \mathrm{C}$ NMR.

The calorimetric measurements reported were carried out at a $10 \mathrm{~K} / \mathrm{min}$ heating (or cooling) rate in a Mettler Star ${ }^{e}$ DSC System; the temperature scale was calibrated using n-octane, indium, and

Table 1

Characteristics of the copolymers used.

\begin{tabular}{llll}
\hline Polymer & $M(\mathrm{PtBa})$ & $M(\mathrm{PS})$ & $M_{\mathrm{w}} / M_{\mathrm{n}}$ \\
\hline PtBa & 327,000 & - & 1.14 \\
P305 & 201,800 & 206,200 & 1.20 \\
P307 & 489,000 & 236,600 & 1.13 \\
PS & - & 220,000 & 1.10 \\
\hline
\end{tabular}

tin, and the $C_{\mathrm{p}}$ scale was calibrated by measuring a sapphire standard Two samples were measured for each polymer, and three different runs were done for the $C_{p}$ measurements. The typical amount of polymer sample used was $10 \mathrm{mg}$.

The dielectric experiments were done using both an HP-4284A bridge and a Solartron 1260 gain-phase analyzer with a Chelsea dielectric interface. The samples $(0.25 \mathrm{~mm}$ thickness and $30 \mathrm{~mm}$ in diameter) were introduced in a home-made parallel-plate capacitor and kept under vacuum for two days at room temperature, and afterwards at $383 \mathrm{~K}$ in order to erase the previous thermal history. A home-built cryostat-thermostat was used, which allowed the temperature to be constant within $\pm 0.2 \mathrm{~K}$ at $200 \mathrm{~K}$, and $\pm 0.1 \mathrm{~K}$ above $250 \mathrm{~K}$. The global linear-frequency window available to our experiments is $10^{-2}-10^{6} \mathrm{~Hz}$. All the measurements were carried out in the isothermal mode.

The dynamic-mechanical experiments were carried out on a DMTA V apparatus from Rheometric Scientific. We have chosen the double cantilever geometry, and the experiments were carried out in the isothermal mode, which allowed the temperature to be constant within $\pm 0.2 \mathrm{~K}$ at room temperature. The measurements were done in the frequency range $0.01 \leq f / \mathrm{Hz} \leq 100$. The probes were pressed in a Teflon mould under vacuum, and the temperature was kept $10^{\circ}$ above the $T_{\mathrm{g}}$ of PS during $10 \mathrm{~h}$ in order to erase any thermal history. Afterwards the samples were allowed to reach room temperature while still under vacuum. Two probes were measured for each sample.

The Molecular Dynamic Simulation (MDS) study of Poly(tertbutylacrylate) (PtBa) chains was carried out on a PtBa homopolymer and (PS)-b-PtBA diblock copolymers with different PtBa chain lengths for $P(307)$ and $P(305)$. Three different sequences were chosen to represent P305, P307 and PtBA (see Scheme 1a-c, respectively), and then ten polymer fragments of each sequence were contained in a box under periodic boundary condition (see Scheme 2). Dynamics of 1 ns using Consistent Valbond Force Field (CVFF) [11] for three boxes was run in the temperature range $1000-1700 \mathrm{~K}$. A conformational analysis was done to estimate the variations of the free energy between the conformations of dihedral angles $\phi_{1}$ and $\phi_{2}$ of the samples (see Scheme 1).

\section{Results}

Fig. 1a shows the thermograms obtained for the different samples. The values of the $T_{\mathrm{g}} \mathrm{s}$ are reported in Table 2 . As it can be observed the values of the $T_{\mathrm{g}} \mathrm{s}$ of the copolymers are coincident with those of the homopolymers within the width of the transition (see Fig. 1b for the definition of $\Delta T$ ), which confirms the immiscibility of the two blocks. It has to be noted that the value of $\Delta T$ for the PtBa blocks is much higher in the copolymers than in the PtBa homopolymer, which might be explained in terms of the existence of concentration fluctuations in the copolymers. The values of $\Delta T$ for the PS blocks are similar to those of the PtBa ones. No differences are found for $\Delta T$ of the PS blocks in the copolymers and in the PS homopolymers.

Fig. 2 shows the imaginary part of the permittivity as a function of $T$ and $f$ (the linear frequency) for one of the copolymers in the temperature range of the segmental relaxation (the results for the other copolymers are similar). As it can be observed, at the highest temperatures (but below the $T_{\mathrm{g}}$ of the PS blocks) a strong conductivity contribution is found at low frequencies together with a low intensity process which has been attributed to the structural or $\alpha$-relaxation of the amorphous PtBa chains. As $T$ is decreased the $\alpha$-process moves toward lower frequencies, and a less intense subvitreous $\beta$-relaxation appears. Fig. $2 \mathrm{c}$ shows the $\beta$-relaxation for PtBa and P305 at $208 \mathrm{~K}$. It can be observed that the transition in the copolymer is shifted toward lower frequencies. The corresponding curve for P307 is not shown because the maximum is outside the experimental frequency window, 


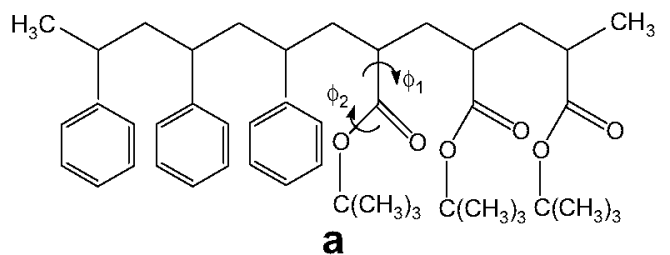

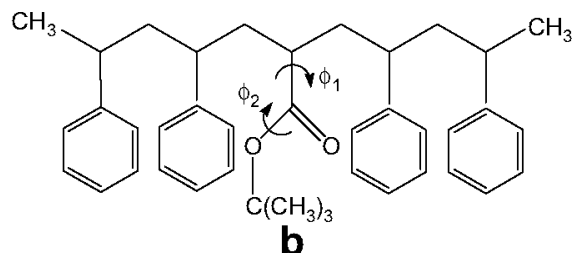<smiles>CC(CC(CC(CC(CC(C)C(=O)OC(C)(C)C)C(=O)OC(C)(C)C)C(=O)OC(C)(C)C)C(=O)OC(C)(C)C)C(=O)OC(C)(C)C</smiles>

Scheme 1. Model structures for (a) P(305); (b) $\mathrm{P}(307)$; and (c) PtBa.

and thus it has not been possible to obtain reliable values for the relaxation times. Similar results were obtained for the $\alpha$-relaxation of the other samples.

The relaxation curves have been fitted to a sum of $n$ contributions

$\varepsilon_{\text {dipolar }} *(\nu)=\varepsilon_{\infty}+\left(\frac{\sigma_{\mathrm{dc}}}{i \varepsilon_{0} 2 \pi f}\right)^{s}+\sum_{i=1}^{n} \rightleftarrows \frac{\Delta \varepsilon_{i}}{\left[1+\left(i 2 \pi f \tau_{i, \mathrm{HN}}\right)^{\hat{o}_{i}}\right]^{\gamma_{i}}}$

where the first term of the right hand side (r.h.s.) represents the high-frequency limit of the dielectric permittivity, the second term accounts for the ionic conductivity found at high temperature (see Fig. $2 \mathrm{~b}), \sigma_{\mathrm{dc}}$ being the direct current conductivity, and $\varepsilon_{0}$ is the dielectric permittivity of the vacuum. The exponent $s \leq 1$ takes into account the complexity of the conductivity processes that appear in the low-frequency region. For the simplest ohmic process $s=1$. The last term of the r.h.s. of Eq. (1) describes for the contributions of the segmental (or $\alpha$-) relaxation and of the subvitreous (or $\beta$-) relaxation. For the last two contributions we have used a HavriliakNegami function, in which $\Delta \varepsilon_{i}$ is the intensity of the relaxation, $\tau_{i, \mathrm{HN}}$ is a characteristic time, and the parameters $\delta_{i}$, and $\gamma_{i}$ are dimensionless parameters that describe the symmetric and asymmetric broadening of the loss function $\varepsilon^{\prime \prime}(\nu)$, respectively. They are constrained such as $0<\delta, \delta \gamma \leq 1$. We have found that for the three samples studied the $\beta$-relaxation can be described with a Cole-Cole function, i.e. $\gamma=1$. The fits are the lines in Fig. 2. Fig. $2 \mathrm{~b}$ shows that the d.c. conductivity contribution can be described with $s=1$.

Fig. 3 shows the imaginary part of the elastic modulus of one of the samples at temperatures close to the $\alpha$-relaxation of the PtBa blocks. Physical aging experiments of Shelby and Wilkes have suggested the existence of a weak $\beta$ process in PS This might overlap with the $\alpha$ process of the PtBa blocks, thus leading to an increase of the width and height of the relaxation peak. Fig. 4 shows the same magnitude for temperatures close to the $\alpha$-relaxation of the PS block in the P305 copolymer, similar results were found for the two other samples. Two facts are clearly visible: On one side the scattering of the data appears to be larger than for those of Fig. 3. On the other hand, for some temperatures, the existence of more than one relaxation appears to be clear. Fig. 5 shows that it is possible to build a master curve with the DMTA results of one of the copolymers near the $T_{\mathrm{g}}$ of the PtBa block (similar results were found for the other samples, but not for the results near the $T_{\mathrm{g}}$ of the PS block). This means that from the mechanical point of view the PtBa blocks can be considered as thermorheologically simple materials, and that the shape of the $E^{\prime \prime}(f)$ curves does not change with $T$. Similar results were obtained by Alig et al. for PS-b-poly(isoprene) copolymers However, it was not possible to build a similar master curve with the dielectric results since, as it will be discussed below, the shape of the $\varepsilon^{\prime \prime}(f)$ curves is a function of $T$. The shift factor $a_{T}$ of the frequency axis needed for building the master curves for the three samples (for the $\alpha$-relaxation of the PtBa block) has been fitted to a Williams-Landel-Ferry equation

$\log _{10} a_{T}=-\frac{C_{1}\left(T-T_{0}\right)}{C_{2}+T-T_{0}}$

The constants $C_{i}$ and $T_{0}$ are given in Table 3. However, one cannot discard the possibility that the good results obtained in building the

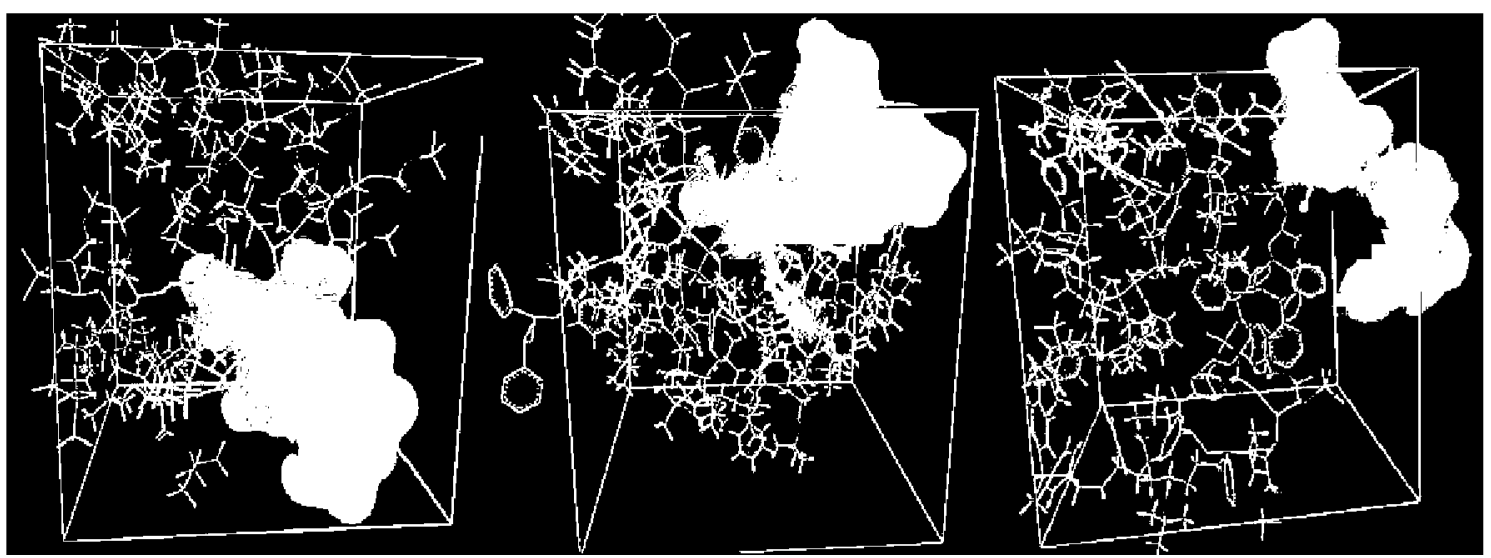

Scheme 2. Representation of boxes for MDS in periodic boundary conditions using Consistent Valbond Force Field (CVFF). 
a

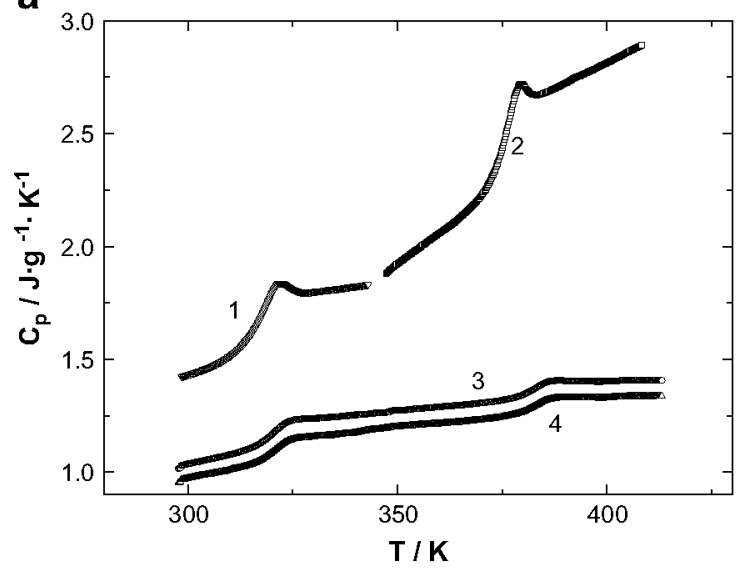

b

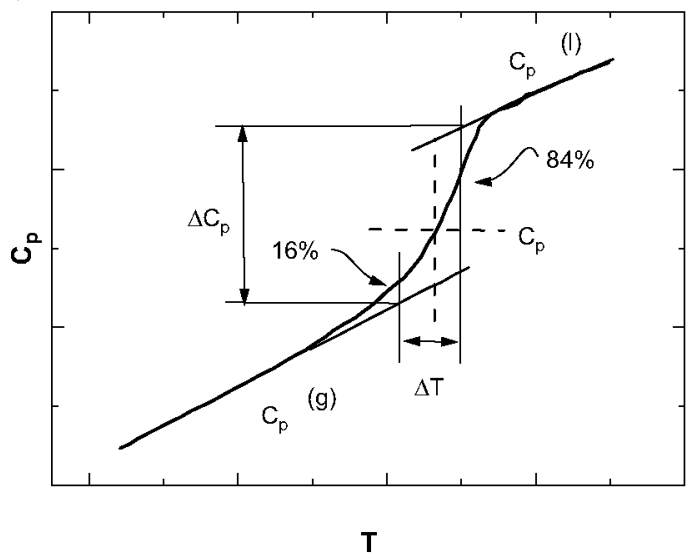

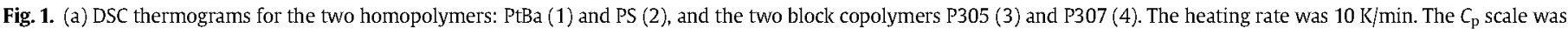
calibrated with a sapphire standard. (b) Construction used to calculate the size of the cooperative rearranging regions according to

master curve are, at least in part, due to the limited frequency range of the DMTA experiments.

The variation of conformational free energy $\Delta G$ obtained from conformational analysis, and activation energy $E_{\mathrm{A}}$ obtained from dielectric experiments is shown in Table 4. Fig. 10 exemplifies the frequency distribution of the conformational change of the dihedral angles $\phi_{1}$ and $\phi_{2}$ (see Scheme 1), obtained from the trajectory of the MDS carried out at $1700 \mathrm{~K}$ for the PtBa homopolymer.

\section{Discussion}

A key concept in the fluctuation approach to the $\alpha$-relaxation is that of the cooperatively rearranging region (CRR) [16], defined as the smallest volume element that can relax to a new configuration independently of the neighboring regions. According to Donth et al. [17] the volume of a CRR is given by

$V_{\alpha}(\delta T, T)=\xi_{\alpha}^{3}(\delta T, T)=\frac{k_{\mathrm{B}} T^{2} \Delta\left(\frac{1}{C_{\mathrm{V}}}\right)}{\rho(\delta T)^{2}}$

where $\rho$ is the density, and usually $C_{\mathrm{v}}$ is approximated by $C_{\mathrm{p}}$. $\delta T=\Delta T / 2,5$, with $\Delta T$ obtained from the $C_{p}$ curves as indicated in Fig. 1b, The values calculated from the DSC experiments according to Fig. 1b are given in Table 2 . The values of $\xi_{\alpha}$ found in the case of the homopolymers are slightly larger than for the block copolymers, although the differences between the samples are close to the calculated uncertainties. These values are similar to those previously reported for other polymers The fact that $\xi_{\alpha}$ is slightly smaller for the block copolymers than for the homopolymers may be due to the microphase separated morphology of the latter. It has been already demonstrated in semicrystalline

Table 2

Parameters' characteristic of the DSC traces of the different polymers in the glass transition region. The definitions of some of the variables are given in Fig. 1b according to Ref. [11]. $\xi_{\alpha}$ is the radius of cooperativity rearranging regions calculated from $\mathrm{Eq}$. (3).

\begin{tabular}{|c|c|c|c|c|c|c|}
\hline \multirow[t]{2}{*}{ Property } & \multirow[t]{2}{*}{ PtBa } & \multirow[t]{2}{*}{ PS } & \multicolumn{2}{|l|}{ P305 } & \multicolumn{2}{|l|}{ P307 } \\
\hline & & & PtBA & PS & PtBA & PS \\
\hline$\overline{T_{g} / \mathrm{K}}$ & 317 & 378 & 319 & 381 & 320 & 382 \\
\hline$\Delta T / \mathrm{K}$ & 3 & 6 & 7 & 6 & 7 & 6 \\
\hline$\Delta C_{\mathrm{p}} / \mathrm{Jg}^{-1} \mathrm{~K}^{-1}$ & 0.21 & 0.29 & 0.15 & 0.085 & 0.13 & 0.082 \\
\hline$C_{\mathrm{p}} / \mathrm{Jg}^{-1} \mathrm{~K}^{-1}$ & 1.69 & 2.52 & 1.16 & 1.36 & 1.09 & 1.29 \\
\hline$\xi_{\alpha} / \mathrm{nm}$ & $3.8 \pm 0.4$ & $3.1 \pm 0.4$ & $2.7 \pm 0.5$ & $2.5 \pm 0.3$ & $2.7 \pm 0.5$ & $2.4 \pm 0.4$ \\
\hline
\end{tabular}

polymers than when the amorphous phase is subject to geometrical constraints the size of the CRR decreases In fact Hong et al. found that there is a correlation between $\xi_{\alpha}$ and the interlamellar separation In the PS-b-PtBa copolymers, the amorphous PtBa phase is constrained by the glassy PS phase.

We have calculated the retardation times characteristic of the $\alpha$-and $\beta$-relaxations for the PtBa blocks from the fits of the dielectric relaxation spectra to Eq. (1). In order to compare the characteristic times of the dielectric and mechanical experiments one has to consider that $E^{*}$ is a modulus while $\varepsilon^{*}$ is a compliance. As a consequence the times obtained from $E^{*}$ are relaxation times, while those from $\varepsilon^{*}$ are retardation times. Richert and coworkers have recently discussed the relationship between the average relaxation, $\tau_{\mathrm{M}}$, and retardation times, $\tau_{\varepsilon}$, obtained from $\varepsilon^{*}$ and from the dielectric modulus $M^{*}=1 / \varepsilon^{*}$. For a Debye-like relaxation they concluded that $\tau_{\varepsilon} / \tau_{\mathrm{M}}=\varepsilon_{0} / \varepsilon_{\infty}$, where $\varepsilon_{0}$ and $\varepsilon_{\infty}$ are the low- and high-frequency limits of the dielectric constant for the relaxation under study

For a relaxation described by the Havriliak-Negami equation no closed expression exists for $\tau_{\varepsilon} / \tau_{\mathrm{M}}$. However, the expression corresponding for the Debye case is an upper limit for the ration of the relaxation and retardation times. We have calculated $\tau_{M}$ from the average retardation times calculated from the fits to Eq. (1). The temperature dependence of the retardation times has been described by Eq. (2), and the corresponding constants are given in Table 3. The relaxation map shown in Fig. 6 points out that the difference between the two copolymers and the PtBa homopolymer in the $\alpha$-relaxation is rather small, almost within the scattering of the data. This means that there is almost no mixing effect at the segmental level, as expected in the strong segregation regime However, clear differences were found for the times corresponding to the $\beta$-relaxation, the relaxation times of the copolymers being larger than those of the homopolymer. These results are unexpected because the $\beta$-relaxation has a more local character than the $\alpha$-one, and contrast with the results found for other copolymers The results found cannot be attributed to any possible coupling between the different contributions in Eq. (1). In effect, as it can be observed in Fig. 2a, there are temperatures for which the different samples present only contribution from the $\alpha$-relaxation, and thus it is possible to determine the relaxation time without any perturbation from the $\beta$-or the conductivity contributions. Fig. 6 also includes the temperature dependence of $\tau_{\alpha}$ calculated from the DMTA experiments using Eq. (2) and the parameters collected in Table 3 for PtBa and P307 (the results for P305 are intermediate and are not shown for the sake of clarity). The figure shows that there is a reasonable agreement between the temperature dependencies predicted by 
a

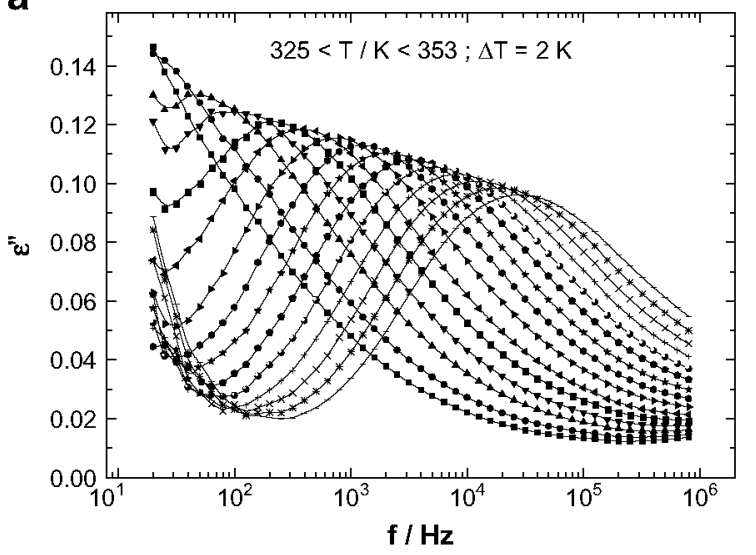

b

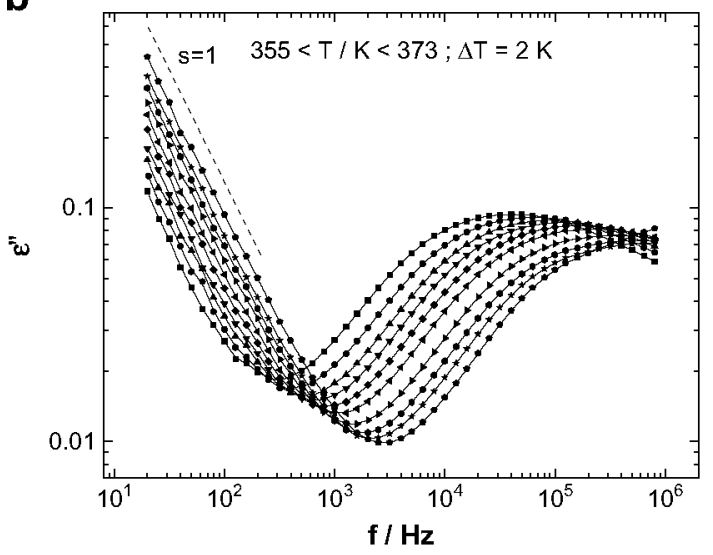

C

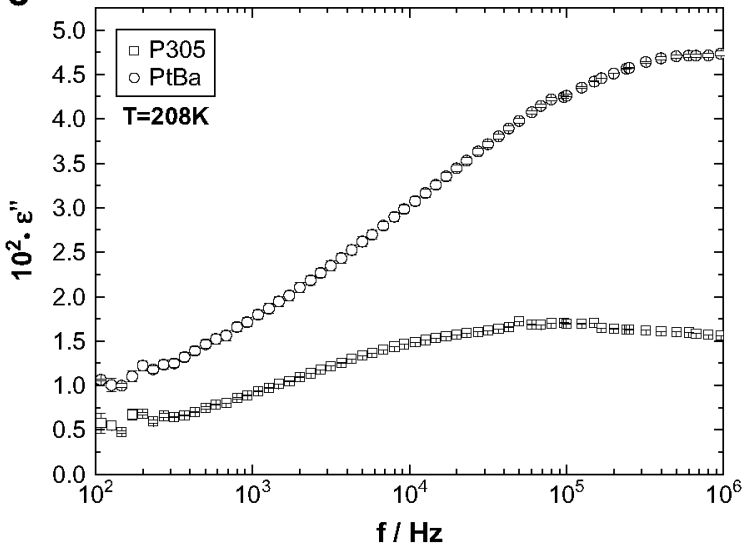

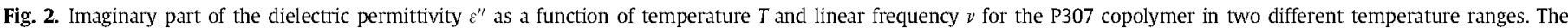

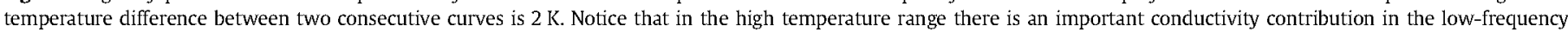
range. The curves are the fits to Eq. (1).

both techniques. This is further confirmed by the fragility parameter $m$ shown in Table 3, which is quite similar to those reported for copolymers of poly(oxyethylene) and poly(oxypropylene)

Nevertheless, the DMTA technique leads to slightly different curves for the two copolymers and the homopolymer. The DMTA

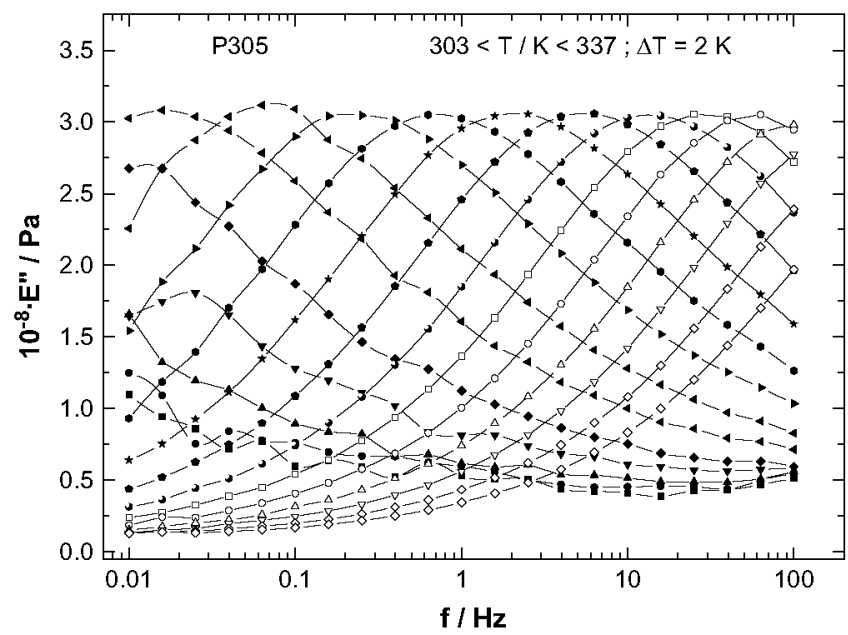

Fig. 3. Imaginary part of the Young modulus $E^{\prime \prime}$ of the P305 copolymer as a function of temperature $T$ and angular frequency $\omega$ in the glass transition region of the PtBa blocks. The temperature difference between two consecutive curves is $2 \mathrm{~K}$. Lines are aid for the eyes. results indicate that the relaxation times of the $\alpha$-relaxation of PtBa are larger than those of the copolymers, which is unexpected because in the latter case the PtBa chains are linked to the glassy PS blocks. It must be stressed that Fig. 6 only compares the

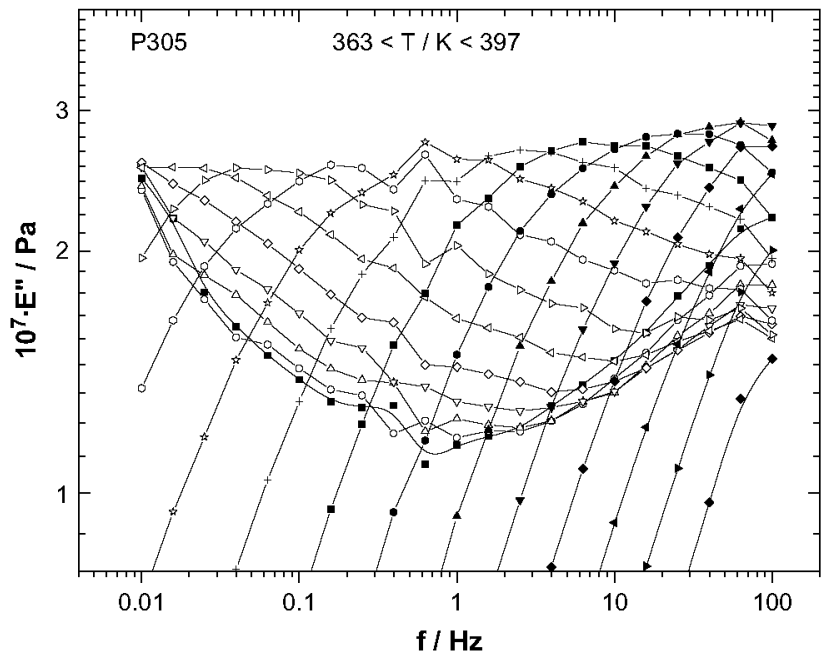

Fig. 4. Imaginary part of the Young modulus $E^{\prime \prime}$ as a function of temperature $T$ and angular frequency $\omega$ in the glass transition region of the PS blocks for the P305 copolymer. The temperature difference between two consecutive curves is $2 \mathrm{~K}$. Lines are aid for the eyes. 

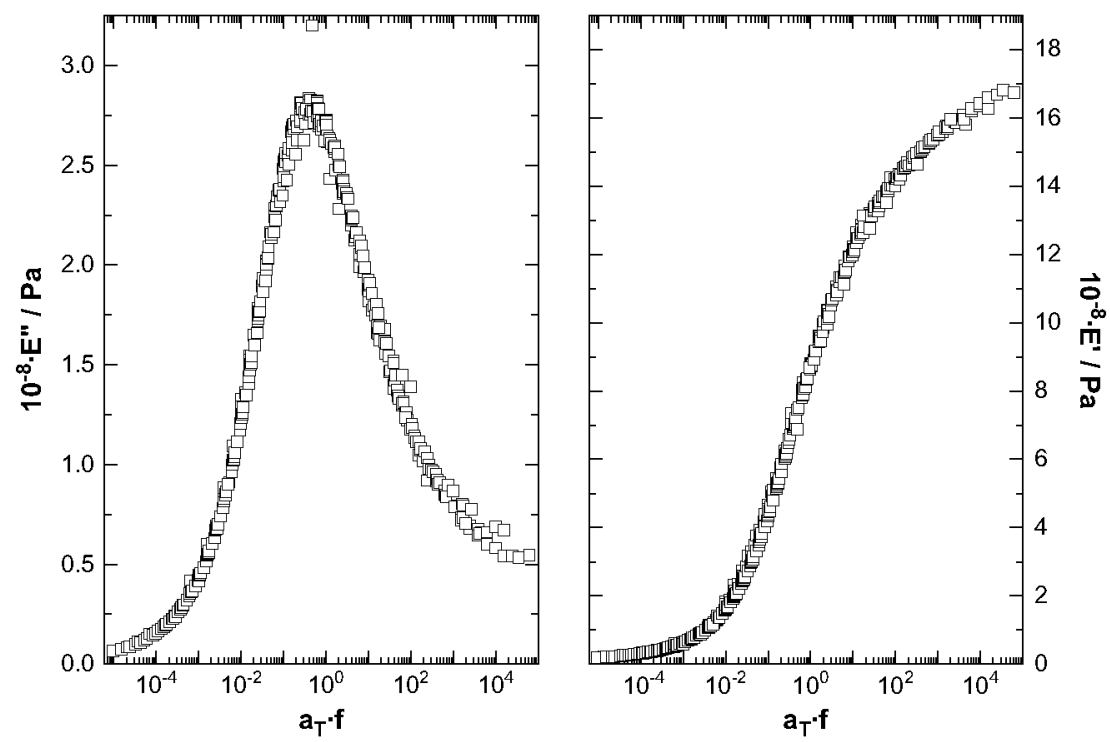

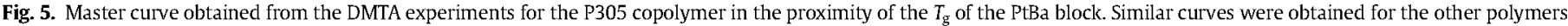
and also in the region close to the $T_{\mathrm{g}}$ of the PS block. $a_{T}$ is the shift factor.

temperature dependencies of $\tau_{\varepsilon, \alpha}$ obtained by both techniques, while the absolute values of $\tau_{\mathrm{M}, \alpha}$ obtained by DMTA are higher than those obtained by dielectric relaxation (around 10 times higher), which is the usual behaviour This is more clearly pointed out in Fig. 7 which shows two examples of the relaxation function calculated from dielectric and DMTA experiments as

$\phi(t)=\frac{\int_{-\infty}^{\infty} H(\ln \tau) \exp [-(t / \tau)] \mathrm{d} t}{\int_{-\infty}^{\infty} H(\ln \tau) \mathrm{d} t}$

where $H(\ln \tau)$ is the relaxation spectra obtained from the $M^{*}$ and $E^{*}$ data using a regularization algorithm. Contrary to the algorithm proposed by Weese the one described by Dominguez-Espinosa et al. allows one to include the low-frequency conductivity contribution in the kernel of the regularization method. Therefore, it is not necessary to extract such contribution from the raw $\varepsilon^{\prime \prime}$ data before proceeding to calculate the retardation spectrum. As $T$ increases, the difference between the relaxation functions of PtBa and the copolymers increases, in accordance with Fig. 6. However, the differences between the $\phi(t) s$ calculated from the electrical modulus remain almost constant over the whole temperature range. The discrepancies found between $\tau_{\varepsilon}$ and $\tau_{M}$ are not new; similar differences were already reported by Havriliak and by Ngai Richert and coworkers

It must be stressed that in the work of the data of the mechanical moduli

Table 3

Parameters of the WLF equation (Eq. (2)) used to build the master curves from the DMTA data in the region close to the $T_{\mathrm{s}}$ of the PtBa block, and those obtained from the relaxation times calculated from the dielectric experiments using the relationship $\tau_{\varepsilon} / \tau_{M}=\varepsilon_{0} / \varepsilon_{\infty}$ from $\quad \tau_{\varepsilon}$ being the retardation time and $\tau_{M}$ the corresponding relaxation time. $m$ is the fragility parameter

\begin{tabular}{lcccc}
\hline Polymer & $C_{1}$ & \multicolumn{1}{c}{$C_{2} / \mathrm{K}$} & \multicolumn{1}{c}{$T_{0} / \mathrm{K}$} & $m$ \\
\hline \multicolumn{2}{l}{ Relaxation times from dielectric experiments } & & \\
PtBA & $11.9 \pm 0.2$ & $42 \pm 3$ & $315.4 \pm 0.7$ & $69 \pm 1$ \\
P305 & $15.7 \pm 0.4$ & $50 \pm 4$ & $308.5 \pm 0.9$ & $74 \pm 2$ \\
P307 & $16.8 \pm 0.3$ & $37 \pm 4$ & $300.7 \pm 0.8$ & $71 \pm 1$ \\
Relaxation times from DMTA experiments & & \\
PtBA & $11.3 \pm 0.4$ & $44 \pm 1$ & $315.4 \pm 0.6$ & $62 \pm 1$ \\
P305 & $15.4 \pm 0.2$ & $66.4 \pm 0.8$ & $319.7 \pm 0.4$ & $69 \pm 1$ \\
P307 & $17.5 \pm 0.3$ & $75 \pm 1$ & $313.6 \pm 0.7$ & $72 \pm 2$ \\
\hline
\end{tabular}

were compared to the corresponding compliances (or alternatively the electrical moduli to the corresponding permittivities), whereas in the present work electrical and mechanical moduli have been compared.

The results shown allow one to test an empirical relation proposed by Alvarez between the parameters of the Havriliak-Negami function and the exponent $\beta$ of the stretchedexponential relaxation function for the structural relaxation [19]: $\gamma \delta=\beta^{1.23}$. We have fitted the master curves of the DMTA experiments to the stretched-exponential function:

$\frac{E^{*}(2 \pi f, T)-E_{\mathrm{u}}}{E_{\mathrm{u}}-E_{\mathrm{r}}}=\int_{0}^{\infty} \frac{\mathrm{d} \phi(t)}{\mathrm{d} t} \exp (-i 2 \pi f \cdot t) \mathrm{d} t$

with $\phi(t)=A \exp \left[-\left(t / \tau_{\mathrm{KWw}}\right)^{\beta}\right], E_{\mathrm{u}}$ and $E_{\mathrm{r}}$ being the unrelaxed and relaxed values of the real part of $E^{*}$, respectively. The results lead to $\beta=0.40,0.36$, and 0.34 for $\mathrm{PtBa}, \mathrm{P} 305$ and $\mathrm{P} 307$, respectively. Similar values have been reported for other poly(acrylate) polymers: 0.37 for poly(methyl acrylate) from DMTA measurements and 0.47 and 0.38 for poly(n-butyl acrylate) and poly(n-hexyl acrylate), respectively The product $(\gamma \delta)^{1 / 1.23}$ at $340 \mathrm{~K}$ takes the values $0.39,0.36$, and 0.33 , respectively, which is in agreement with the empirical relation proposed by Alvarez et al. However, there is an important difference between the description of the data using the $\mathrm{H}-\mathrm{N}$ and the stretched-exponential functions. In effect, the exponent $\beta$ controls both the width of the relaxation and the temperature dependence of the relaxation time whereas the parameters $\gamma$ and $\delta$ are only related to the width of the relaxation.

The intensity of the $\alpha$-relaxation $T \Delta \varepsilon_{\alpha}$ was found to increase slightly with $T$ for the two copolymers over the whole temperature range (see Fig. 8), while it decreases more strongly with increasing

Table 4

Activations energy $\left(E_{\mathrm{A}}\right)$ obtained from dielectric experiments for the $\beta$-relaxations, see Fig. 6 , and variation of conformational free energy $(\Delta G)$ obtained from theoretical result, Eq. (7).

\begin{tabular}{lllr}
\hline Polymer & \multicolumn{1}{c}{ PtBa } & $\mathrm{P}(307)$ & $\mathrm{P}(305)$ \\
\hline $10^{-3} \cdot E_{\mathrm{A}} / R$ & $1.45 \pm 0.04$ & $1.32 \pm 0.05$ & $1.1 \pm 0.1$ \\
$10^{-3} \cdot \Delta G / R$ & $-1.6 \pm 0.1$ & $-1.9 \pm 0.1$ & $-2.2 \pm 0.2$ \\
\hline
\end{tabular}




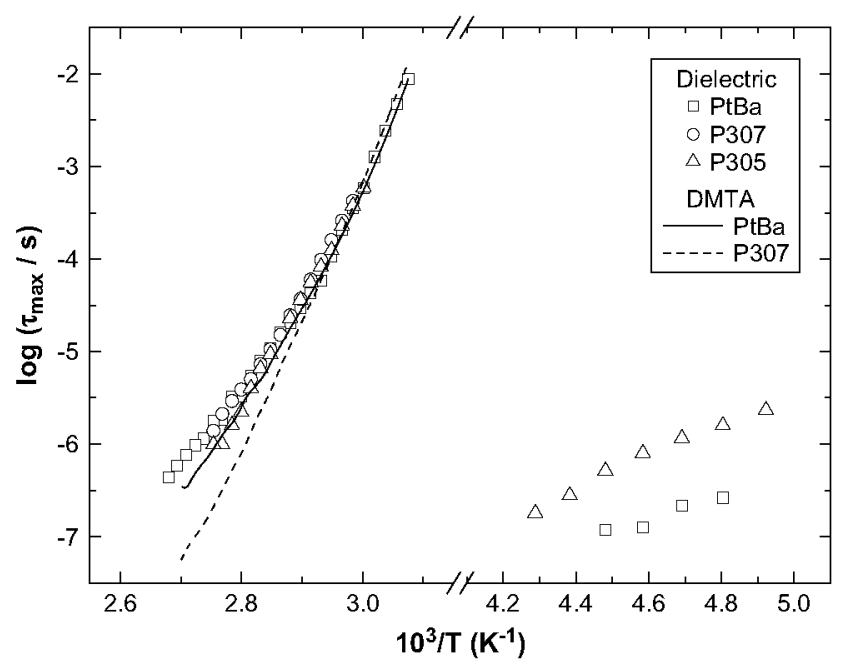

Fig. 6. Relaxation map obtained for the PtBa blocks in the different samples. The symbols correspond to relaxation times $\tau_{\mathrm{M}}$ calculated according to $\tau_{\varepsilon} / \tau_{\mathrm{M}}=\varepsilon_{0} / \varepsilon_{\infty}$ from the retardation times $\tau_{\varepsilon}$ corresponding to the maximum of the loss dielectric curves. The two curves have been calculated from the shift factors of the DMTA experiments for PtBa and P307. The curve corresponding to P305 is intermediate to those shown and has not been plotted for the sake of clarity.

$T$ for the homopolymer as in most of the polymers

It must be stressed that the product $T \Delta \varepsilon_{\alpha}$ for the homopolymer is $3-4$ times larger than for the copolymers. Taking into account that the molecular weight of the PtBa blocks is similar, the big difference in the relaxation intensity must be due to the existence of frozen amorphous PtBa blocks due to the existence of glassy PS regions in the microphase separated copolymers. This has already been mentioned to explain that the size of the CRR is smaller in the block copolymers than in the homopolymers. One might expect that such constraints should be released as $T$ is increased The intensity of the $\beta$-relaxation was found to slightly increase with $T$, although in this case the change for the PtBa homopolymers is less pronounced. Although the intensity of the $\beta$-relaxation is slightly larger for the homopolymers than for the copolymers, the differences never exceed $30 \%$

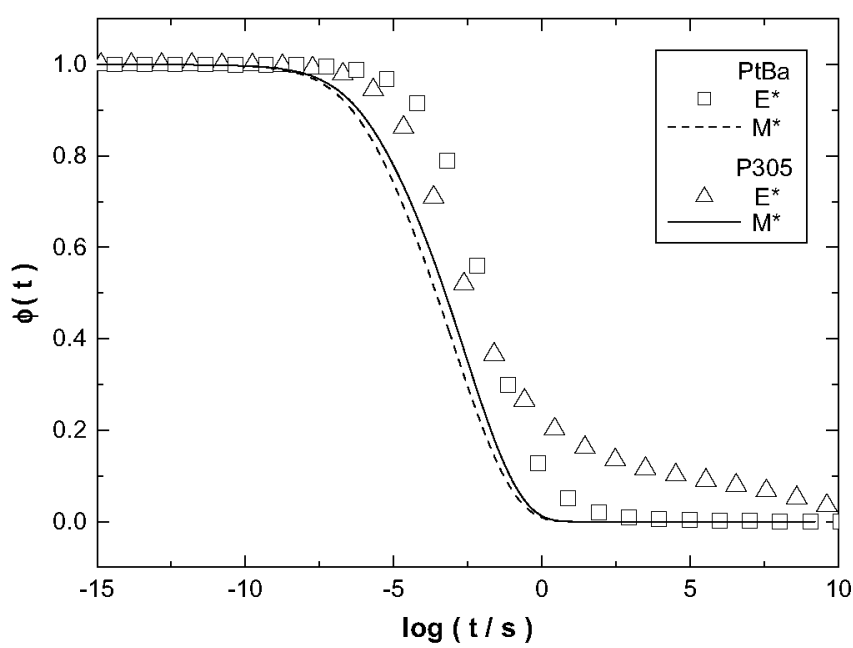

Fig. 7. Relaxation curves calculated from the dielectric and DMTA experiments for two samples at a temperature in which both experiments overlap: $333 \mathrm{~K}$ for PtBa and $331 \mathrm{~K}$ for P305. The curves corresponding to dielectric experiments were calculated from the dielectric modulus representation.

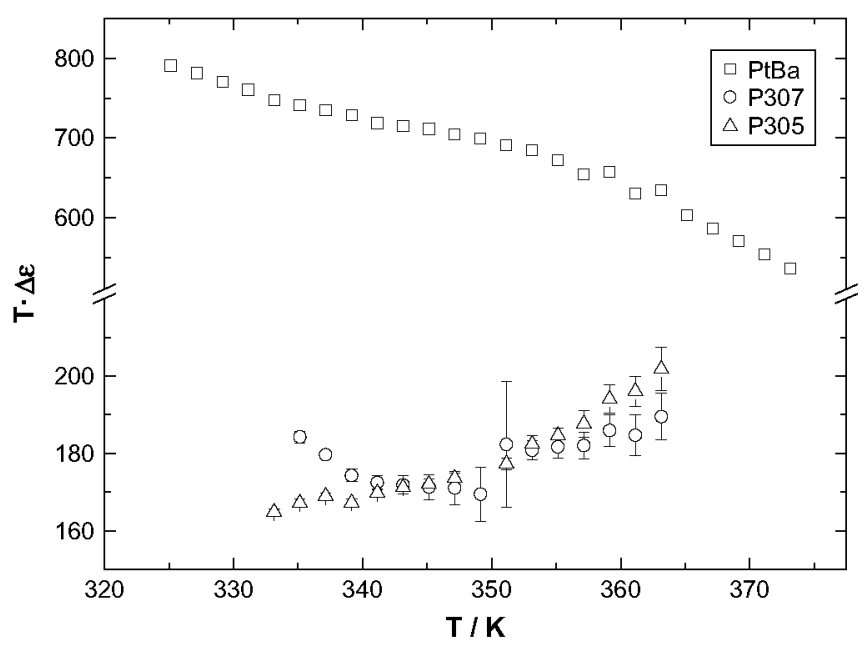

Fig. 8. Temperature dependence of the strength of the $\alpha$-transition obtained from the dielectric experiments. The uncertainty for the PtBa homopolymers is within the size of the symbols.

Fig. 9 shows the width of the relaxation calculated

$$
\begin{aligned}
w_{\mathrm{H}-\mathrm{N}}= & \frac{1}{\delta} \log \left\{\frac{\left[2+\cos \left(\delta^{\pi} / 2\right)\right]+\sqrt{\left[2+\cos \left(\delta^{\pi} / 2\right)\right]^{2}-1}}{\left[2+\cos \left(\delta^{\pi} / 2\right)\right]-\sqrt{\left[2+\cos \left(\delta^{\pi} / 2\right)\right]^{2}-1}}\right\} \\
& \times\left[1+7 \frac{\ln \gamma}{17}\right]^{-\sqrt{\delta}}
\end{aligned}
$$

where $\delta$ and $\gamma$ are the parameters of the Havriliak-Negami function, and $w_{\mathrm{H}-\mathrm{N}}$ is the width of the relaxation, which for the case of a Debye relaxation $(\delta=\gamma=1)$ corresponds to 1.14 decades. $w_{\mathrm{H}-\mathrm{N}}$ increases with $T$ for the three polymers studied, the width being larger for the copolymers except near $T_{\mathrm{g}}$. A small increase of the width of the relaxation has been also reported for poly(methyl acrylate) although in this case some the results might be due in part to the proximity of the $\beta$-relaxation. As already mentioned, the change of $w_{\mathrm{H}-\mathrm{N}}$ with $T$ is responsible of the fact that no master curve can be built with the dielectric data, which contrasts with the DMTA results. In the case of the $\beta$-relaxation, $w_{\mathrm{N}-\mathrm{H}}$ increases with $T$ as usual, and is slightly broader for the copolymers than for the homopolymer.

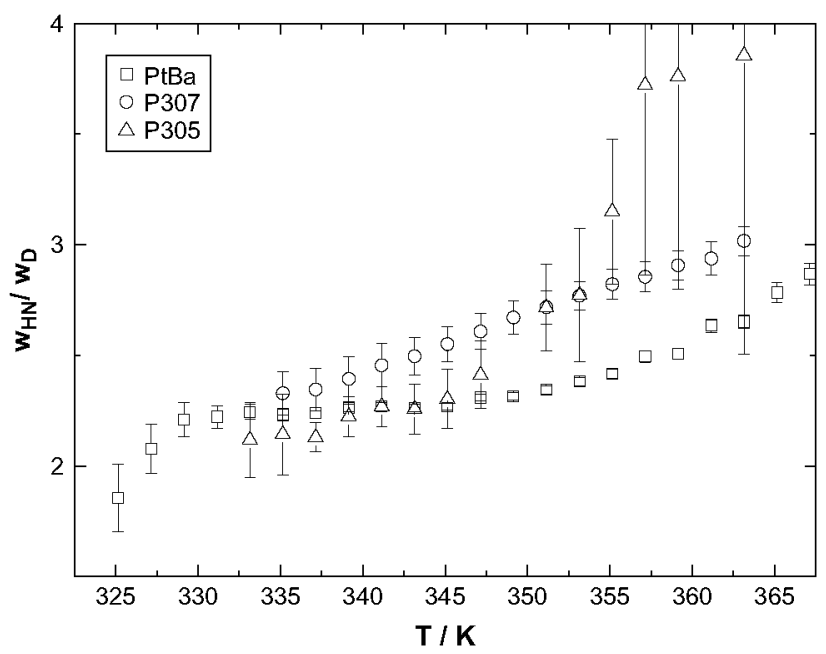

Fig. 9. Temperature dependence of the width $w_{\mathrm{H}-\mathrm{N}}$ of $\alpha$-relaxation of the PtBa blocks obtained from the dielectric experiments. $w_{D}$ refers to the width of a Debye relaxation (1.14 decades). 
Moynihan and Schroeder have related the mean square deviation of $\ln \tau_{i}$ from its average, $\left\langle\Delta^{2} \ln \tau\right\rangle$, to the volume of the CRR by

$\left\langle\Delta^{2} \ln \tau\right\rangle=\left[\frac{B T_{2}}{\left(T-T_{2}\right)^{2}}\right]^{2} \frac{k_{\mathrm{B}} v}{\Delta C_{\mathrm{p}} V_{\alpha}}$

where $B$ and $T_{2}$ are the parameters that appear in the Vogel-Fulcher equation

$\ln \frac{\tau}{\tau_{0}}=\frac{B}{T-T_{2}}$

and $v$ is the specific volume.

A similar equation was found for $\left\langle\Delta^{2} \ln \tau\right\rangle$ in terms of the FreeVolume theory:

$$
\left\langle\Delta^{2} \ln \tau\right\rangle=\left[\frac{B T_{2}}{\left(T-T_{2}\right)^{2}}\right]^{2} \frac{k_{\mathrm{B}} T \Delta \kappa}{(\Delta \alpha)^{2} V_{\mathrm{FV}}}
$$

where $\Delta \kappa$ and $\Delta \alpha$ are the changes in isothermal compressibility and isobaric expansivity at the glass transition, respectively. $T_{2}$ is now the temperature at which the free-volume of the equilibrium liquid would vanish. Taking into consideration typical values of the Prigogine-Defay ratio $\prod=\Delta C_{\mathrm{p}} \Delta \kappa / T v(\Delta \alpha)^{2}$, Moynihan and Schroeder [36] found that $V_{\mathrm{FV}}^{1 / 3} / V_{\alpha}^{1 / 3} \approx 1.8-2.4$.

$\left\langle\Delta^{2} \ln \tau\right\rangle$ is a measure of the width of the distribution of relaxation times that can be directly related to the $\beta$ parameter of the KWW function [37]. It can also be calculated from the relaxation spectra obtained from the dielectric modulus [26,27]. This method leads to the following values for $\left\langle\Delta^{2} \ln \tau\right\rangle: 2.4,6.0$, and 4.2 for PtBa, P305 and P307, respectively. These results can be compared with those provided by Eq. (7) in order to check the consistency of the dielectric data with the size of the CRR obtained by DSC. Using the data of the relaxation map and Eq. (8) we have obtained the parameters $B$ and $T_{2}$ for the copolymers and for PtBa. Assuming a value of $v$ similar for the three samples, and using the data of Table 2 we have calculated the following values of $\left\langle\Delta^{2} \ln \tau\right\rangle$ in the $T_{\mathrm{g}}$ region of the PtBa blocks: $2.9,7.8$, and 5.5 for PtBa, P305 and $\mathrm{P} 307$, respectively. These values are slightly larger than those obtained from the dielectric relaxation experiments, although they follow the same order for the different samples. However, it must be stressed that both Eqs. (7) and (9) are consistent with an increase of $\left\langle\Delta^{2} \ln \tau\right\rangle$ as $T$ is decreased, which is contrary to what it has been found for the present systems (see Fig. 9).

Ngai has proposed an empirical correlation between the $\beta$ parameter and the value of the relaxation time of the $\beta$ transition at $T=T_{\mathrm{g}}$, with $T_{\mathrm{g}}$ defined as the temperature at which the relaxation time of the $\alpha$-transition is $10^{4} \mathrm{~s}$. We have found that the correlation leads to values which are almost 3 decades higher than the values shown in Fig. 6. In an analysis of random copolymers of PS and poly(n-butyl acrylate) Ngai has indicated that these kinds of discrepancies may arise from the concentration fluctuations in the copolymers Furthermore, it must be considered that Ngai's correlation was proposed for the so-called Johari-Goldstein $(J-G)$ secondary relaxations. It is not straightforward to test whether the $\beta$-transition of the present samples fulfil the conditions for the J-G relaxations pointed out by Ngai and Paluch because the $\alpha$-and $\beta$ - relaxations cannot be measured at the same temperature within the experimental frequency regime. This would be important because one of the conditions of the $J-G$ relaxations is that they do not follow an Arrhenius-like temperature dependence above $T_{\mathrm{g}}$. The other condition is that the $\mathrm{J}-\mathrm{G}$ relaxation is highly pressure dependent.

Using MSA techniques, a conformational analysis using MDS was performed in order to clarify the apparent anomalous behaviour of the variations detected in the differences on the relaxation times of the local $\beta$ transition. To reach an appropriate explanation our hypothesis is centered in terms of conformational free energy differences of the ester group of the tert-butyl moiety on the block copolymer in comparison with that of the homopolymer. The conformational analysis of the variation of the angles $\phi_{1}$ and $\phi_{2}$ (see Scheme 1), is the starting point for this study.

For this reason we have considered two structural models (see Scheme 1) for the conformational analysis of the copolymers in which we assume that the tert-butyl group is in between of two styrene groups and the other with the tert-butyl group surrounded by tert-butyl moieties and only one styrene segment (see Scheme 1a). Although the first assumption is not a realistic model, it should be useful to know which could be the effect of the conformational behaviour of the tert-butyl moiety on the whole response of the copolymer from relaxational point of view.

The relaxation maps obtained from dielectric relaxation experiments show that for the $\beta$-transition the characteristic time for the PtBa blocks is faster in the homopolymer than in the copolymers, see Fig. 6 . This is an unexpected result because the $\beta$-relaxations have a more local character. We assume that the relaxations times for PtBa blocks of the $\mathrm{P}(307)$ and $\mathrm{P}(305)$ are being affected for the PS blocks. The free energy $(\Delta G)$ for the conformational change of the dihedral angles $\phi_{1}$ and $\phi_{2}$ of the PtBa block, was evaluated through the Boltzmann equation

$\Delta G-R T \ln \frac{f \phi_{1}}{f \phi_{2}}$

where $f \phi_{i}$ is the frequency at which a given value of $\phi_{i}$ appears.

From these MDSs it is possible to obtain the distribution of frequencies of the conformational changes. This distribution is represented as an example of the general behaviour in Fig. 10 for the homopolymer fragment. The frequencies of the dihedral angles $\phi_{1}$ and $\phi_{2}$ of PtBa block, for each system were analyzed. The conformational free energy $(\Delta G)$ was obtained from the plots of $\ln \left(f \phi_{1} / f \phi_{2}\right)$ against $1 / T$. The conformational analysis was used as a tool to elucidate the degree of mobility of the tert-butyl group of the different models. By this way the variations of the conformational free energy decrease in the following way: PtBa $>\mathrm{P}(307)>\mathrm{P}(305)$. Table 4 summarizes the activation energy values for the $\beta$-relaxation from dielectric measurements, and also the free energy of the conformational change estimated by MDS. As can be observed the conformational change $\Delta G$ is more favourable (more negative) for $\mathrm{P}(305)$. Therefore in this case there is a larger mobility of the ester

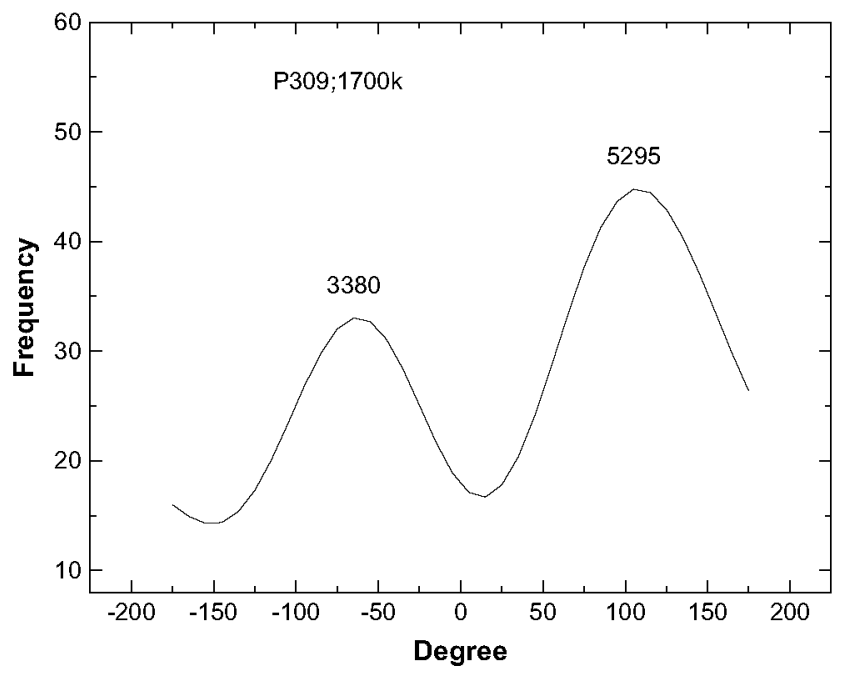

Fig. 10. Distribution of frequencies of the dihedral angles $\phi_{1}$ and $\phi_{2}$ for the model PtBa homopolymer at $1700 \mathrm{~K}$. 
group, and then it this possible to assume that the relaxational process should be less hindered than in the other samples. These results could explain the relaxational behaviour of the homo and copolymers, i.e. as the mobility of the ester group is more favourable the activation energy for the $\beta$-relaxation decreases.

\section{Conclusions}

A calorimetric, dielectric, and the dynamic-mechanical study of PtBa and two diblock copolymers of PS and PtBa has been carried out around the glass transition temperature of the systems. Two separate $T_{\mathrm{g}} \mathrm{s}$ are present in each of the copolymer which are very close to those of the corresponding homopolymers. The size of the cooperative rearranging regions for each type of block has been calculated from the $C_{\mathrm{p}}$ vs. $T$ curves, and it shows no significant differences between the samples.

The relaxation map for the PtBa blocks has been calculated from the dielectric relaxation curves. While no significant differences have been found between the results of the different samples for the $\alpha$-relaxation, the relaxation times corresponding to the $\beta$ relaxation are smaller for the PtBa homopolymer than for the copolymers. This is a surprising result because the motions corresponding to the $\beta$-relaxation have a more local character than those of the $\alpha$-one. The temperature dependence of the relaxation time obtained from the DMTA experiments for the $\alpha$-relaxation is very similar to the one obtained from dielectric relaxation. However, the magnitude of the relaxation times is different, the values from DMTA being larger than those from dielectric modulus. Moreover, the DMTA experiments show that there are some differences between the temperature dependence of the relaxation times of the three samples. The width of the dielectric relaxation curves increases with $T$, which is unusual.

Conformational analysis seems to be a powerful tool to describe the differences in the mobility of the ester group. The results point out that the mobility of the t-butyl acrylate group is strongly influenced by its molecular surrounding, thus leading to different activation energies in the homopolymer than in the copolymers. This is in qualitative agreement with the fact that the relaxation times of the $\beta$ transitions are different in both types of chains.

\section{Acknowledgments}

This work was supported in part by MCyT under grants FIS200612281-C02-01 and INTERFASES CAM-S-505-MAT-227. L.G. and D.R. thank to Fondecyt projects 1080007 and 1080026 for partial financial support. C.S. thanks to Fondecyt for a post-doctoral fellowship.

\section{References}

Hamley IW. The physics of block copolymers. Oxford, England: Oxford University Press; 1998.

Kotaka T, Adachi K. Macromol Symp 1997;3:124.

Alig I, Kremer F, Fytas G, Roovers J. Macromolecules 1992;25:5277.

Karatasos K, Anastasiadis SH, Floudas G, Fytas G, Pispas S, Hadjichristidis H, et al. Macromolecules 1996;29:1326.

Kyritsis A, Pisis P, Mai S-M, Booth C. Macromolecules 2000;33:4581.

Vogt S, Gerharz G, Fisher EW, Fytas G. Macromolecules 1992;25:5986.

Moreno S, Rubio RG. Macromolecules 2002;35:5483.

Ma L, He Ch, Suzuki T, Azuma M, Bin Y, Kurosu H, et al. Macromolecules 2003;36:8056.

Pakula T. In: Kremer F, Schönhals A, editors. Broadband dielectric spectroscopy. Berlin: Springer-Verlag; 2003.

de Buhr J, Riesen R, Widmann J, Jörimann U. Mettler User Com. 7, Mettler Toledo $\mathrm{GmbH}$, Analytical; 1998.

Maple J, Hwang M, Jalkanen K, Stockfish T, Hagler A. J Comput Chem 1998; 19:430.

Korus J, Hempel E, Beiner M, Kahle S, Donth E. Acta Polym 1997;48:369.

Shelby MD, Wilkes GL. Polymer 1998;26:6767.

Alig I, Floudas G, Avgeropoulos A, Hadjichristidis N. Macromolecules 1997;30: 5004.

Riande E, Díaz-Calleja R, Prolongo MG, Masegosa RM, Salom C. Polymer viscoelasticity. Stress and strain in practice. New York: Marcel Dekker; 2000. Donth EJ. Relaxation and thermodynamics in polymers. Berlin: Akademie Verlag; 1992.

Donth E, Hempel E, Schick C. J Phys Condens Matter 2000;12:L281.

Moreno S, Rubio RG, Luengo G, Ortega F, Prolongo MG. Eur Phys J E 2001; $4: 173$.

Chamberlain RV. Phys Rev Lett 1999;82:2520.

Tran TA, Saïd S, Grohens Y. Composites Part A Appl Sci Manuf 2005;36:461. Hong PD, Chuang WT, Yeh WJ, Lin TL. Polymer 2002;43:6879.

Ito N, Richert R. J Chem Phys 2005;123:106110.

Jäckle J, Richert R. Phys Rev E 2008;77:031201.

Havriliak S, Havriliak SJ. Dielectric and mechanical relaxation in materials. Munich: Hanser Verlag; 1997.

Ngai KL, Mashimo S, Fytas G. Macromolecules 1988;21:3030.

Weese J. Comput Phys Commun 1993;77:429.

Domínguez-Espinosa G, Díaz-Calleja R, Riande E, Gargallo L, Radic D. J Chem Phys 2005; 123:114904.

Alvarez F, Alegría A, Colmenero J. Phys Rev B 1991;44:7306.

Sanchis A, Prolongo MG, Masegosa RM, Rubio RG. Macromolecules $1995 ; 28: 2693$.

Ngai KL. Macromolecules 1999;32:7140.

Colmenero J, Alegria A, Santangelo PG, Ngai KL, Roland CM. Macromolecules $1994 ; 27: 407$.

Sanchis MJ, Díaz-Calleja R, Pelissou O, Gargallo L, Radiç D. Polymer 2004;45: 1845.

Kanapitsas A, Pissis P, Kotsilkova R. J Non-Cryst Solids 2002;305:204

Richert R, Stickel F, Fee RS, Maroncelli M. Chem Phys Lett 1994;229:302.

Schönhals A. In: Kremer F, Schönhals A, editors. Broadband dielectric spectroscopy. Berlin: Springer-Verlag; 2003.

Moynihan CT, Schroeder J. J Non-Cryst Solids 1993;160:52.

Moynihan CT, Boesch LP, Leberge NL. Phys Chem Glasses 1973;14:122.

Ngai KL, Paluch M. J Chem Phys 2004;120:857.

Kollman P. Chem Rev 1993;93:2395.

Jorgensen W. Acc Chem Res 1989;22:184.

Gilson M, Given J, Bush B, McCammon J. Biophys J 1997;72:1047.

Gilson M, Given J, Head M. Chem Biol 1997;4:87. 